\title{
Essay
}

\section{More than a Picture: Helping Undergraduates Learn to Communicate through Scientific Images}

\author{
Fiona L. Watson and Barbara Lom
}

\author{
Department of Biology and Program in Neuroscience, Davidson College, Davidson, NC 28035-7118
}

Submitted July 3, 2007; Accepted September 19, 2007

Monitoring Editor: Jeffrey Hardin

\begin{abstract}
Images are powerful means of communicating scientific results; a strong image can underscore an experimental result more effectively than any words, whereas a poor image can readily undermine a result or conclusion. Developmental biologists rely extensively on images to compare normal versus abnormal development and communicate their results. Most undergraduate lab science courses do not actively teach students skills to communicate effectively through images. To meet this need, we developed a series of image portfolio assignments and imaging workshops in our Developmental Biology course to encourage students to develop communication skills using images. The improvements in their images over the course of the semester were striking, and on anonymous course evaluations, $73 \%$ of students listed imaging skills as the most important skill or concept they learned in the course. The image literacy skills acquired through simple lab assignments and in-class workshops appeared to stimulate confidence in the student's own evaluations of current scientific literature to assess research conclusions. In this essay, we discuss our experiences and methodology teaching undergraduates the basic criteria involved in generating images that communicate scientific content and provide a road map for integrating this curriculum into any upper-level biology laboratory course.
\end{abstract}

\section{INTRODUCTION}

Developmental biologists have historically described the processes by which organisms develop and grow using drawings, sketches, and, more recently, photographs and digital images. Although modern developmental biologists have many experimental tools at their disposal, much of the scientific data collected fundamentally rely on comparing observations between an organism's normal (control) and abnormal development. For instance, when scientists genetically manipulate organisms to exhibit either a loss- or gainof-function for a particular gene, they visually compare the mutant with the control to identify developmental abnormalities. In fact, quantitative measurements are often collected directly from digital images. Images are powerful means of both generating and communicating scientific results; a compelling image can convey a scientific result more effectively than words, whereas a poor image can readily undermine a result or interpretation. A scientist's abilities to evaluate and generate high-quality scientific images are crit-

DOI: $10.1187 /$ cbe. $07-07-0045$

Address correspondence to: Barbara Lom (balom@davidson.edu). ical to success. It is therefore incumbent upon educators to train future scientists to be able to evaluate results from images presented in the literature and generate high-quality scientific images to communicate their own data. Our motivation for training students in imaging and visually based analytical skills arose from previous experiences where strong student research results were too often undermined by sloppy figures. We found most students come to our Developmental Biology class with no prior instruction in generating scientific images or constructing figures and that few students intuitively know how to communicate effectively with pictures. In our experience, even advanced undergraduate biology majors with strong laboratory experience, graphing, and scientific writing skills have not had opportunities to develop visual communication skills as part of their training.

Upper-level undergraduate biology courses that include a laboratory component provide the ideal setting for helping students develop the skills for generating high-quality scientific images that effectively communicate scientific results. Fortunately, we found that undergraduates readily and eagerly learn digital image acquisition, presentation, and analysis. In fact, our students extended their imaging skills to 
evaluate and critically assess the accuracy of published research conclusions. Although this essay describes our experiences and methods incorporating imaging exercises and assignments into our Developmental Biology course, we have also successfully used this approach in a group investigation lab course in microscopy and believe similar approaches for introducing image literacy skills could also be easily incorporated in other image-rich upper-level biology courses such as Cell Biology, Neurobiology, and Histology.

\section{IMAGE TRAINING CURRICULUM OVERVIEW}

We developed image portfolio and critique exercises within our Developmental Biology course that encourage undergraduates to develop effective communication skills using scientific images. Our Developmental Biology laboratory syllabus was divided into four projects (Figure 1). The laboratory assignments, worth a total of $40 \%$ of the course grade, included two image portfolio assignments (5\% each), one semi-independent research project $(10 \%)$, and one independent research project $(25 \%)$ that included an oral poster presentation and submission of a cover page image. The weighting of these four lab assignments was intentionally lowest at the beginning of the semester to encourage student improvement and raise expectations as their skills mature. In addition to the lab assignments, we integrated one 50-min and two 20-min discussions (referred to as workshops) on specific criteria and examples of clear scientific images into the lecture schedule (Figure 1).

Instead of a written lab report for the two research projects, we asked students to submit their research project reports in the form of scientific posters. We selected the poster format because generating a research poster is an important image-intensive format taught less frequently than scientific manuscript writing. Moreover, generating a research poster helps students understand how to communicate their research at conferences, a step that typically precedes manuscript writing in the scientific research process. In particular, students gain experience in optimizing a poster layout and design, in distilling the essence of the research project's experimental design using bullet points and/or easily understood diagrams, and in learning to present their results using images and graphs. In addition, by orally presenting research posters to their classmates, undergraduates can further develop their oral communication skills.
All laboratory assignments (Figure 1) are submitted electronically in a PowerPoint file (Adobe Systems, San Jose, CA). Electronic submissions enable instructors to assess the quality and resolution of the images, which can be lost during printing. Electronic submissions also allow the instructors to retrieve and project the images during the inclass workshops. (As an important technical aside, the images captured using digital cameras can be large, making PowerPoint presentations unwieldy in size. To reduce the PowerPoint file size without individually decreasing the resolution of each image, save the PowerPoint file as JPEG. PowerPoint will place an image of each slide into a folder and the student can simply create a new PowerPoint presentation by inserting each of these JPEG slides. The size of the file will decrease to about one $\mathrm{MB}$, which can be readily e-mailed.) Students also submit printed copies of their images and posters to facilitate written instructor feedback and posting in the lab. Printed color copies of posters are also compiled in a binder and used as examples of the types of research projects and quality of posters for students in subsequent course offerings.

\section{IMAGE WORKSHOPS AND LABORATORY ASSIGNMENTS}

\section{First Image Portfolio Assignment}

Through laboratory exercises and in-class workshops, we present imaging fundamentals in a manner that minimizes overwhelming students with detail before they become familiar with using microscopes, handling embryos, and acquiring initial images (Figure 1). Although we are fortunate to have digital cameras and software designed specifically for our microscopes, many of the digital cameras on the consumer market are reasonably priced, and inexpensive adaptors that fit into microscope eyepieces can be created or purchased (Clarke, 2007; Martin Microscope, 2007). Freeware image analysis software such as ImageJ (Mac: Softpedia, 2007; others: http:/ / rsb.info.nih.gov/ij/) can be used to measure morphological features, analyze data, and construct figures. A portfolio assignment is a series of digital images that students capture using specific microscopes and/or model organisms. The first portfolio assignment is an exercise for students to learn how to produce digital images through the stereomicroscope. Students generate two scientific images of model organism specimens such as

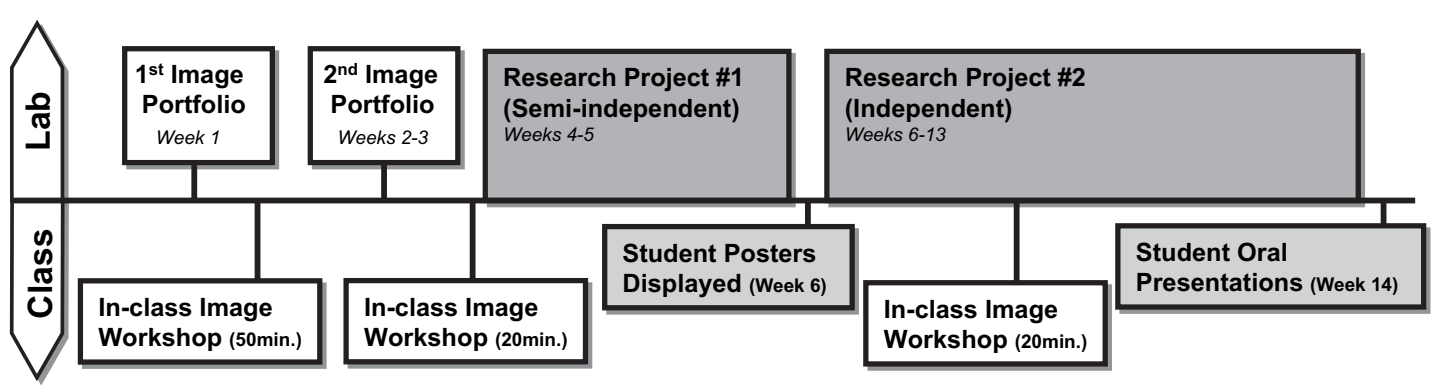

Figure 1. Overview and time sequence of in-class image workshops and laboratory assignments. This diagram illustrates the time and content sequence of classroom and laboratory activities that we used to help students develop and hone the skills required to communicate effectively using images. 
tadpoles, zebrafish embryos, or fruit fly larvae, as well as two familiar "unknown" images. These "unknowns" can be anything a student chooses to place under the microscope, such as a nail file, a penny, or even a dust ball (Figure 2). The familiar unknown component of the assignment gives students a chance to be creative. In addition, we found that by imaging familiar items, students quickly came to understand the magnifying power of the microscopes they will be using throughout the semester. Magnifying a familiar object demonstrates magnification power more readily than magnifying embryos or cells that are not yet familiar. Because there are no references for the scale of specimens imaged through a microscope, students must generate an accurate scale bar for each of the images they include in their portfolio assignments.

For this first portfolio assignment, we intentionally give students minimal guidelines on parameters such as specimen layout, background, and lighting. They focus instead on learning how to handle embryos (fixed and/or live), use the microscope, capture images, and generate a scale bar. We have found that discussions of layout issues can be lost if introduced in the first session when directions for using the microscopes and image capture software are also provided.

\section{First In-Class Image Workshop}

After submission of portfolio 1 images and before the next laboratory session, we devote a 50 -min class period to discussing guidelines and criteria for generating effective scientific images by pointing out the strengths and weaknesses of the images our students have created. This image workshop emphasizes that scientific images are a powerful form of communication that go beyond pretty pictures. Communicating scientific content adds another layer of skill beyond simply capturing an image.
During the workshop, we introduce image criteria divided into two broad categories. The first and most important category includes criteria that must be considered at the time an image is captured, which include the following:

1. identifying the point to be communicated by the image

2. identifying the specific audience(s)

3. determining optimal magnification

4. optimizing resolution

5. adjusting fine focus

6. optimizing lighting

7. reducing any nonspecific or distracting background elements

8. optimizing the signal-to-noise ratio

9. aligning specimens within the frame and to each other

10. verifying that the entire specimen is in the frame and oriented logically (i.e., dorsal side up)

The second category of image criteria includes those criteria that can be modified after the image has been generated, using software such as Photoshop (Adobe Systems, San Jose, $\mathrm{CA})$. This category included the following:

1. appropriate placement, size, contrast, and labeling of the scale bar

2. use of arrows or other symbols to highlight details (if the main result is not immediately evident)

3. use of text to label panels

4. use of all available "real estate" — that is, cropping the image appropriately

Introduction of each of these criteria is accompanied by examples of images from our students and from peer-reviewed journals. The students point out specific strengths and weaknesses of each image and make suggestions to
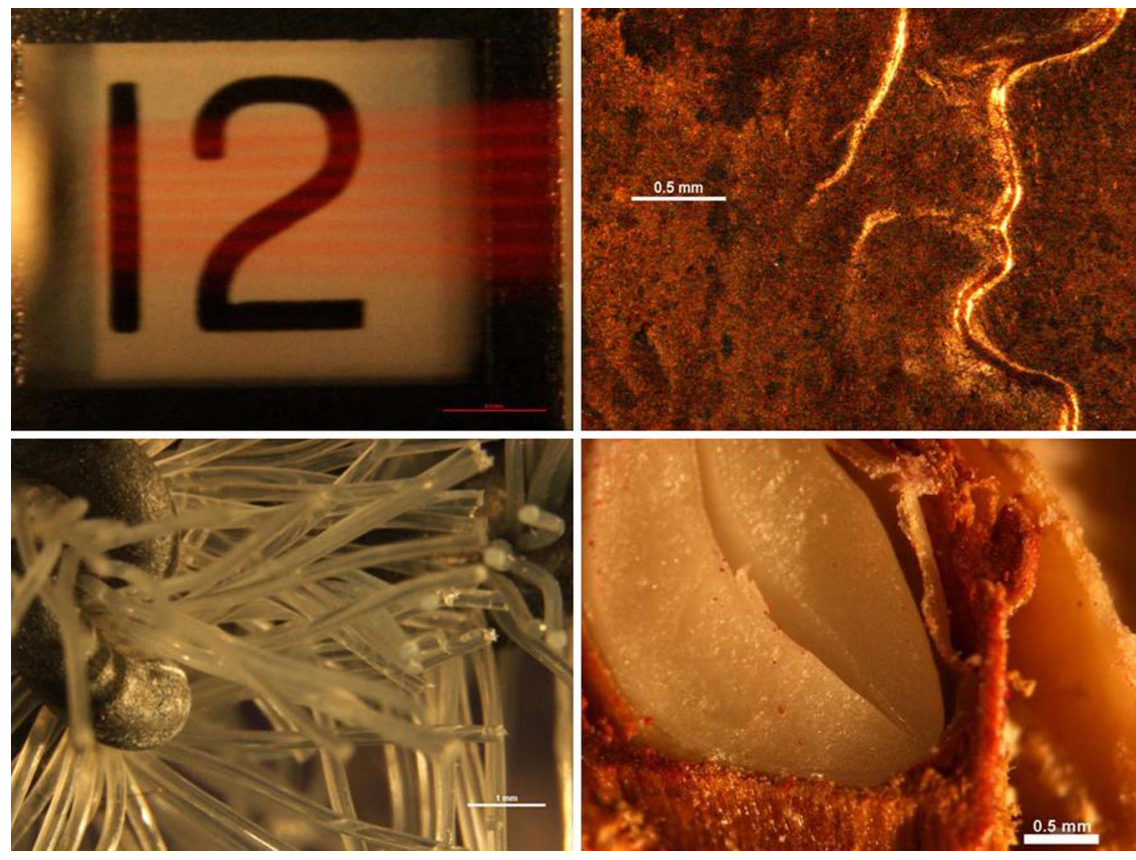

Figure 2. Familiar "unknowns." Examples of student-generated images of familiar unknowns in the first and second image portfolio assignments. These unknowns represent (clockwise from top left): a second hand passing the date on a wristwatch, a penny, a dried apple slice, and a laboratory brush. Note that students commonly had difficulty creating scale bars that were placed and/or labeled properly. 
improve each image. By seeing their own images projected on a large screen in conjunction with examples from the literature, students learn how important it is to optimize elements such as focus, magnification, resolution, and background in order to enhance the message that the image is conveying. During the last few minutes of the workshop, we show the student-generated familiar unknown images (Figure 2). Seeing the broad range of "unknowns" imaged by their peers enables students to appreciate the differences between the information relayed by scientific images versus that of images with more esthetic goals. The unknown images provide an enjoyable and fun way to motivate student creativity and remind students that visually stunning images have an important place in scientific communication (Frankel and Whitesides, 1997; Kramer and Kunkel, 2001; Bourley and Hirsch, 2002; Amato, 2003; Frankel, 2004). Viewing these "unknowns" as a class also provides good opportunities to discuss orientation, lighting, framing, and other esthetic features while priming the pump for the subsequent cover image component of the independent research project. Many of the unknown images are quite striking and creative (in subsequent offerings of the course we hope to create a class calendar). Over the course of the 50 -min discussion we show approximately 90 images. Although the pace may appear rapid, the students learn very quickly to identify the improvements required for their images to meet the criteria for generating effective scientific images.

\section{Second Image Portfolio Assignment}

The second image portfolio assignment is designed to teach students how to use an upright epi-fluorescent microscope and to continue developing the imaging skills learned in the first lab. This second portfolio assignment requires two laboratory periods during which students learn immunostaining, generate two series of overlay images using an upright epi-fluorescence microscope, have opportunities to improve their scientific images submitted in portfolio 1, and generate two additional unknown images using any microscope. Immunostaining is an important technique in developmental biology. We use a primary antibody to a neurofilamentassociated antigen (3A10; Developmental Studies Hybridoma Bank, University of Iowa, Iowa City, IA) with a fluorescent secondary antibody (Alexa 488-conjugated goat anti-mouse; Invitrogen-Molecular Probes, Eugene, OR) to immunostain neurons in frozen sections $(20 \mu \mathrm{m})$ of Xenopus brain and eye. During the longer rinses and incubations, students use the time to become familiar with the upright epi-fluorescence microscope using a prepared triple-labeled slide of cultured cells (Invitrogen-Molecular Probes) that is particularly resistant to photobleaching. Students generate the first series of overlay images by taking an image of this prestained slide using each of the three filter cubes (blue/ green/red) and an accompanying phase image using transmitted light. Students learn how to adjust the microscope's fine focus because the layer of cultured cells on the prepared slide is much thinner than the samples they previously worked with on the stereomicroscope. The images generated using these slides can be used as the basis for a discussion on choices for adjusting the focus and optimizing resolution using slides that are thicker and have a broader depth of field, such as those in their immunostained Xenopus eye and brain sections. In the following lab session, students capture phase and fluorescent images of their own immunostained tissue. Students learn to orient the slide to find appropriate anatomical features and adjust the fine focus to illustrate a specific set of immunostained neurons. With these images of immunostained cells and tissues, students learn to use layers and channels in Photoshop to generate an overlay image and create a figure with multiple panels.

Students generate two more "unknowns" using either the upright or the dissecting microscopes. During the first week of portfolio 2, students learn to use Photoshop (Adobe Systems). We teach the basic skills needed to generate an overlay image including working with layers, multiple channels, and tools for image cropping, adjusting contrast and/or brightness, and importing an appropriate scale bar. The students are very enthusiastic about learning to work with Photoshop and spend a great deal of time outside of the designated lab periods learning to use the program and optimizing their images. We take this opportunity to discuss the kinds of manipulations in Photoshop that are inadmissible when submitting a scientific image and emphasize that they must always keep copies of their original, unmodified images. This discussion is particularly important because of how easy it is to "doctor" an image and how difficult it can be to determine the veracity of data contained within an image. We emphasize that scientists are bound by an honor code to adhere to only admissible manipulations of images. This point could be made further by showing absurd examples from collaborative sites such as Wikipedia and YouTube. Moreover, keeping an original copy of each image is particularly important because it may be critical to return to a unmodified image to examine specific features or revise a figure and also because many journals now require submission of original images in conjunction with submitted manuscripts (Rossner and Yamada, 2004; Pearson 2005; Council of Science Editors, 2006).

\section{Second In-Class Image Workshop}

After submission of the second portfolio assignment, we host a second, shorter in-class image workshop. This discussion emphasizes the criteria for generating a figure composed of multiple image panels, creating a figure layout, and many of the postimage manipulations. Again, we show anonymous examples of student images and ask the students to evaluate and suggest improvements for each image. The improvement between images submitted for the first portfolio and second portfolio is striking (Figure 3), and many students expressed a sense of pride and accomplishment. During this workshop, we discuss the finer details of creating effective scientific images such as use of arrows, frame, orientation, labels, cropping, and the size and location of scale bars (usually in the lower right corner). For figures containing two or more image panels, we discuss scale, labels, specimen orientation, when single versus multiple scale bars are necessary, and the alignment of the image panels within a figure (Figure 4). Many student images show similar errors, so we use two or three student images to emphasize each point, which has the added benefit of showing the students that they are learning together. In our experience, the most common errors include the size and 
placement of the scale bar (Figures 3 and 4), the alignment of image panels within a figure (Figure 4A), and conservative cropping (wasted real estate; Figure 3, A and B). At the end of this workshop, we invite students to improve both image portfolios and resubmit in the next few weeks for a potentially improved grade. Almost $85 \%$ of our students chose to improve and resubmit their image portfolios, and all resubmitted work showed clear improvements that merited higher grades. In particular, we saw marked improvement on scale bars, background, framing of the specimen, and adjustment to the fine focus. We also encourage students to enter their best images in scientific photography competitions (i.e., Chatterjee, 2006; Nikon, 2007; Olympus, 2007).

\section{Third In-Class Image Workshop}

For the third in-class workshop, which occurs after students have begun their second, independent research projects (Figure 1), we show anonymous images from the portfolio resubmissions and introduce the concept of the cover image. Again, we discuss the strengths and weaknesses of each image. Because there are usually very few, if any, improvements to be made to the images, it takes students longer to spot errors. We emphasize that even minor and cosmetic problems within a figure can bring the quality, and hence the validity, of science into question. Some of the common errors at this point in the training include lack of discernable edges for images within a multipanel figure, uneven spacing between panels, more subtle scale bar errors (i.e., presenting multiple scale bars when all panels captured at the same magnification or presenting only a single scale bar for images taken at more than a single magnification), and inappropriate scale bar labels (i.e., $0.005 \mathrm{~mm}$ instead of $5 \mu \mathrm{m}$ ). As an extension, this workshop could also be used as an oppor- tunity to discuss and evaluate video sequences illustrating dynamic developmental events that are frequently published in journals as supplemental material.

\section{Cover Image}

Generating a cover image is a fun way for students to exercise their creativity and further hone their imaging skills (Figure 5). The cover image can be straight science that illustrates a scientific result or concept, but it can also straddle the fields of art and science and have illustrative components that are present purely for esthetic purposes. The cover image assignment is a good opportunity to introduce undergraduates to an emerging field of artistic photomicrography (Davidson and Rill, 2003; Frankel, 1998, 2001). During the second half of the third image workshop, we present a series of cover images along with their descriptive cover captions. We plant the seed that any time a student's manuscript is accepted for publication, s/he should be prepared to submit a striking cover image. In our experience, undergraduates are rarely aware of the prestige a cover image can bring to a scientist or notice the process for submission and selection of cover images. Guidelines for submitting a cover image include three main criteria. First, the image must pertain to the experiment but cannot be an image that appears in the submitted manuscript (or, in our course, the poster). Second, the image must be scientifically sound, i.e., the image must not mislead or overstate any interpretation of the experimental results. Third, the cover image must be accompanied by a short descriptive paragraph written for a broad audience. We emphasize that the cover image can be a synthesis between science and illustration-scientifically sound yet graphically creative and attractive-by showing recent covers of journals such as Development, Developmental
Figure 3. Image portfolio assignments of embryos using a stereomicroscope. A and B each portray a set of three scientific images from two different students' work. Images submitted by both students for the first image portfolio assignment (I), include distracting and disproportionate amounts of background, suboptimal focus, saturation of specimen, inability to frame the entire organism, and/or disproportionately small scale bars. Both students showed dramatic improvements between the first and second portfolios (compare I and II). Some of the errors present in the images submitted for the second image portfolio (II) included improper specimen layout (angle), low contrast between specimen and background, and scale bar errors. Many of the resubmitted images show marked improvements in the background quality, specimen layout, framing, and scale bars (III).
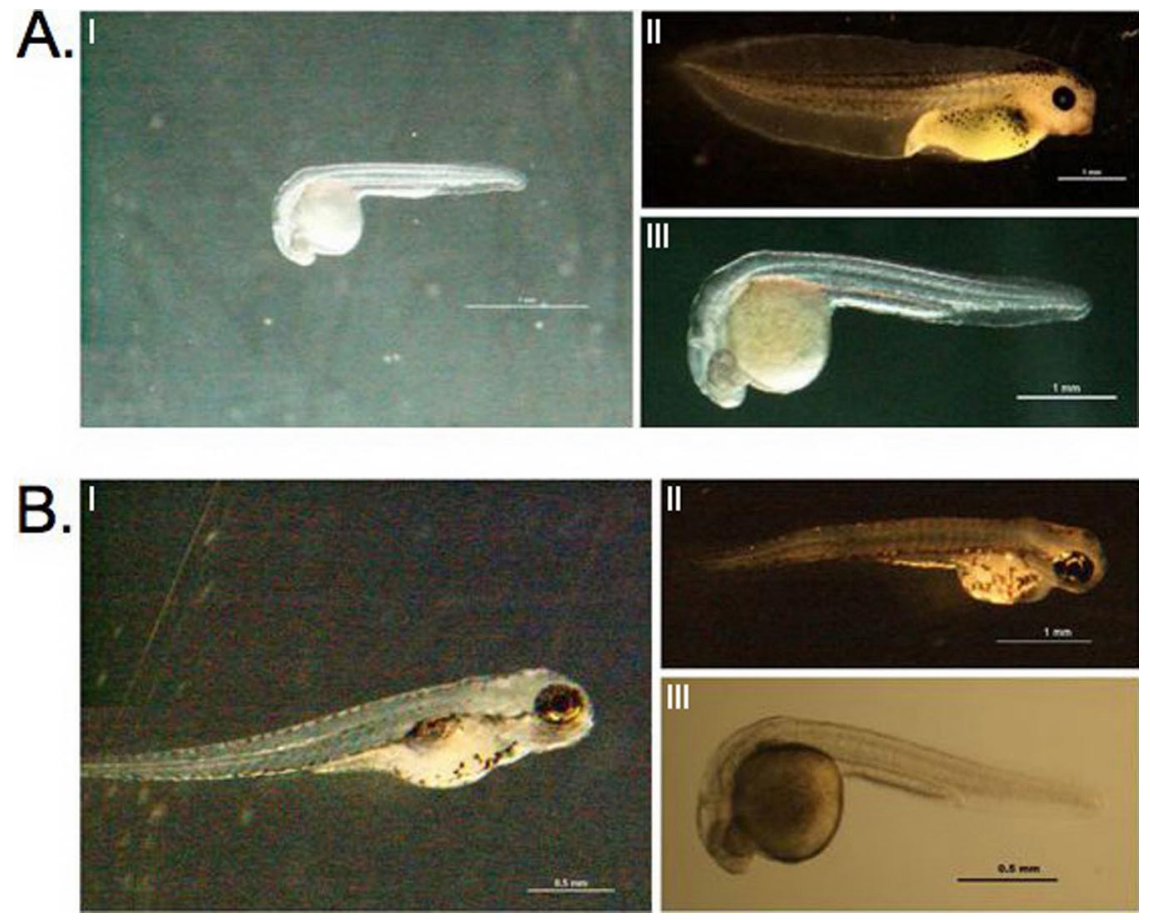
Biology, Cell, Science, and Nature. We were surprised to find that many of our students who prefer rigid guidelines found the task of generating a cover image particularly challenging (Figure 5A). By contrast, those students who enjoy the creative aspects of imaging welcomed the cover image assignment (Figure 5, B and C). Some of the stumbling blocks in generating a cover image were tied to students' inability to grasp the concept and purpose of a cover image as well as their inexperience with journal covers in a world where electronic copies cross their desks more often than print copies.

\section{Research Projects}

Devoting a few weeks in lab to the creation and analysis of scientific images prepares students to start their semi-inde-
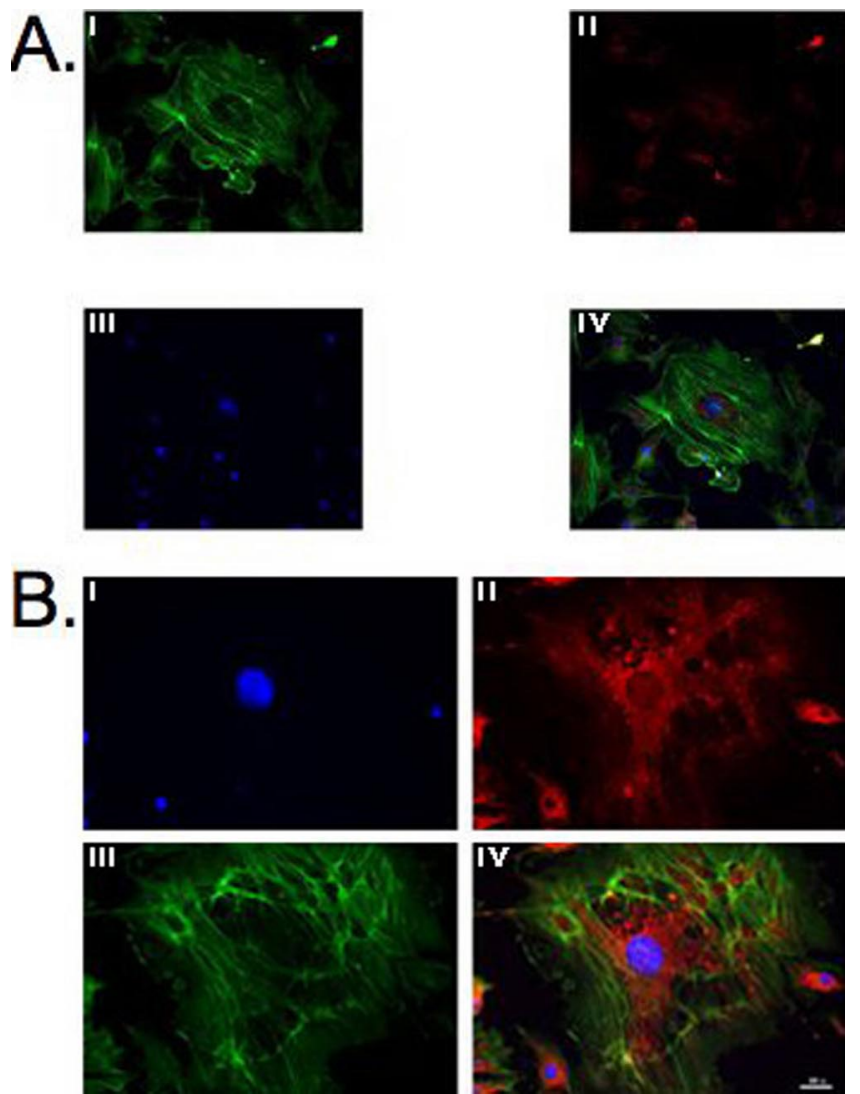

Figure 4. Second image portfolio assignment generated through an epi-fluorescence microscope. A and B represent a series of four images from two different students' work. The series of images is based on a single field of view taken using three different fluorescent channels (I-III) and an overlay of these three images using Photoshop (IV). (A) The unequal spacing, poor contrast, suboptimal focus, absence of scale bar, and distracting saturated cell in the top right hand corner are some of the more noticeable errors. (B) The images show optimization of the fine focus, adequate fluorescent intensity for all three channels, and an appropriately located scale bar (though its label is not clearly visible). Cells are triple-labeled bovine pulmonary artery endothelial cells on a commercially prepared slide (Invitrogen-Molecular Probes): mitochondria are stained with MitoTracker (red); F-actin is labeled with BODIPY FL phallacidin (green), and nuclei are labeled with DAPI (blue). pendent and then independent research projects with greater understanding, familiarity, and confidence in utilizing microscopes to collect and communicate their experimental data. For the first (semi-independent) research project, each student exposes developing Xenopus laevis embryos to a known teratogen (i.e., retinoic acid, ethanol, or the pesticide malathion; Chemotti et al., 2006). Students design their own experiments with respect to teratogen concentration and exposure schedule and individually identify and determine how to measure morphological features that may reveal developmental abnormalities. Students then prepare a scientific poster that contains an introduction, a materials and methods section, a results section that includes at least two high-quality images and two graphs generated from measurements of at least two morphological features, a discussion, a conclusion, and references. Because most students have never created a scientific poster, we encourage them to submit their posters before the deadline to receive preliminary feedback before the posters are graded. We also prepare a binder that contains examples of posters generated by students from previous years (names removed for anonymity) including the instructor's written comments. This feedback allows students to verify their experimental results, interpretations, and image presentation while also stimulating confidence to seek guidance and feedback throughout the research process, a skill particularly important for successfully carrying out the independent research project. Students submit a small $(8.5 \times 11$ inch $)$ color copy of their final semi-independent research poster. These posters are then displayed on a bulletin board in the lab (Figure 6A) so that students can observe their classmates' different styles of presenting similar scientific data and compare outcomes.

For the second (independent) research project, students must research, design, and repeat their own experiments with full control over variables such as treatment(s), exposure time and rates, developmental window, and any other variables using one of many model organisms. The students have approximately $8 \mathrm{wk}$ to carry out their experiments. Because the research projects are often executed outside the formal weekly lab periods, each student e-mailed us a brief weekly progress report (Campbell and Lom, 2006) that frequently revealed students were thinking about image capture, analysis, and presentation throughout the research project. We have found that students often underestimate the time required to analyze the results of their research project; thus we require that they complete all replicate experiments no later than the seventh week. Students orally presented their research posters to the class during the final lab session and submitted their final research poster approximately $1 \mathrm{wk}$ later. The oral poster presentation and the final poster submission deadline were separated to allow students time to incorporate any comments raised by classmates or instructors during the oral poster presentation. For grading purposes, the quality of the images and figures submitted must adhere to the image criteria discussed during both workshops to receive a reasonable grade.

\section{Student Oral Poster Presentation}

At the end of the semester, students present their independent research projects to their classmates by projecting the poster on a large screen and verbally "walking" the class 
through the poster in a manner similar to any scientific research conference (Figure 6B). Projecting the poster on a screen eliminates costs and lead time associated with printing large-format posters. Moreover, because the screen is located at the same height as a poster, students can choose to use their hands or a pointer to indicate specific figures as they discuss their results. To encourage dialog during the presentations, we require student audience members to engage with the presenting student by asking relevant questions, as one would during the oral presentation of the poster at a scientific research conference. Students can then use this valuable feedback to improve their final independent research poster (Figure 6C). We feel that learning to ask questions will make students more comfortable talking to researchers about their work, another important skill for success in the sciences.

A few students voluntarily elected to print their posters in large-format and present them again at the college's annual spring undergraduate research symposium. Both the inclass presentation and the symposium forums provided valuable opportunities for our undergraduates to present their research to their peers, ask questions, and listen to others present their research.

\section{EVALUATION}

In response to the question, "What was the most important skill/concept you learned?" on an anonymous end-of-semester course evaluation, $73 \%$ of our students $(n=16)$ credited the imaging workshops as developing skills that gave them confidence in evaluating research. One student further commented, "Blowing them [images] up and seeing them while we were talking helped me understand things." Ninety-three percent of our students agreed or strongly agreed with the statement, "I appreciated the opportunities to develop imaging skills via the portfolio assignments." One hundred percent of our students agreed or strongly agreed with the following two statements: "The lab experience helped me appreciate the nature of scientific research" and "The lab experience helped me refine my lab skills and achieve expertise." In addition, 93\% of students reported a preference for poster presentations over written lab reports, which corresponded strongly to our preference as instructors. We preferred correcting posters because the students had to learn to distill their work to the most essential images and words in a concise and logical manner that fits the $8.5 \times$ 11-inch format of a mini-poster.
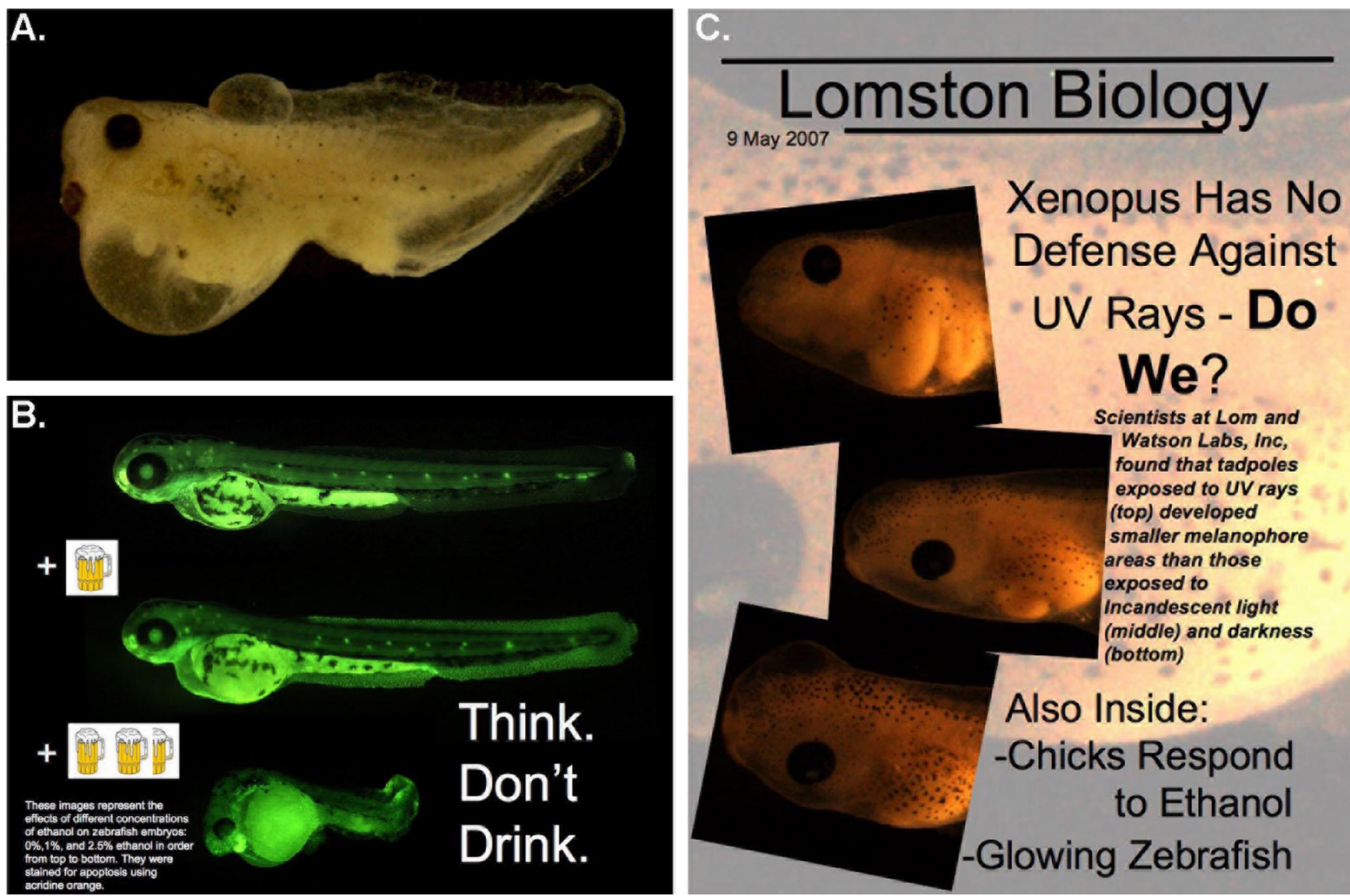

Figure 5. Cover image assignment. (A-C) Examples of covers submitted by three different students illustrate the range of submissions from straightforward (A) to creative (B and C), where even a spoof journal title was created (C). 

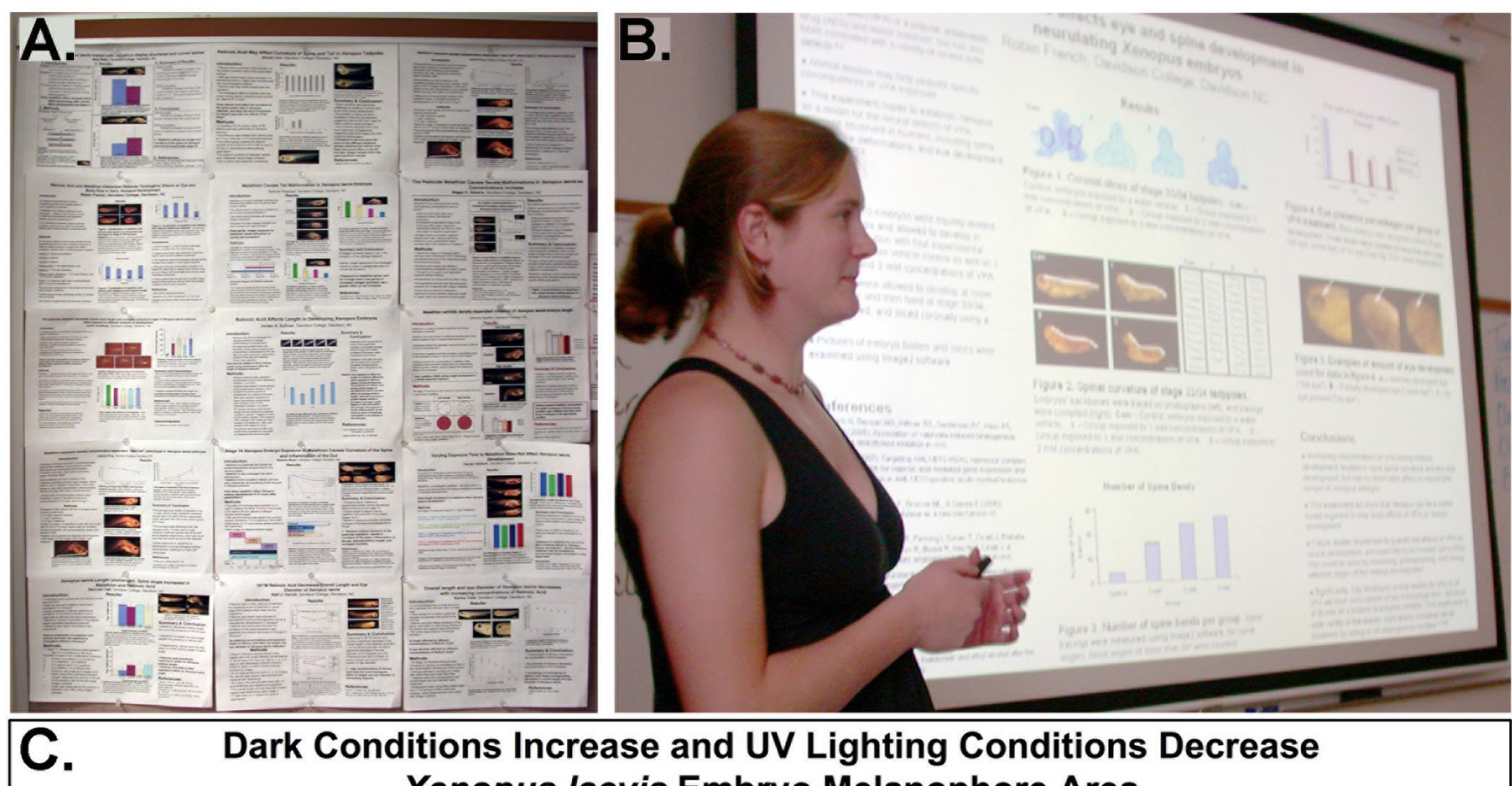

\section{Dark Conditions Increase and UV Lighting Conditions Decrease Xenopus laevis Embryo Melanophore Area}

\section{Introduction}

-Decreasing frog populations worldwide point to global element of change that frogs cannot combat, such as ozone depletion $2,3,5$

-UV light has been linked to decreased hatching rate, smaller size, and anatomical malformations $\mathrm{s}^{3,5,7}$

-Melanophore production is thought to protect from UV damage $\mathrm{e}^{1,3,4,5,6,7,8}$

How does UV light exposure affect Xenopus laevis melanophore size?

\section{Methods}

-108 stage 9 embryos were divided into three groups and raised for three days:

-24 hours of darkness

-12 hours in incandescent light, 12 hours in darkness

.12 hours in UV light (300-400nm), 12 hours in darkness

-Embryos were fixed with $1 \%$ gluteraldehyde,

photographed, blinded, measured for melanophore area on gut with ImageJ software, unblinded, and analyzed with ANOVA

-Total melanophore area determined by multiplying average melanophore size by total number of melanophores on gut
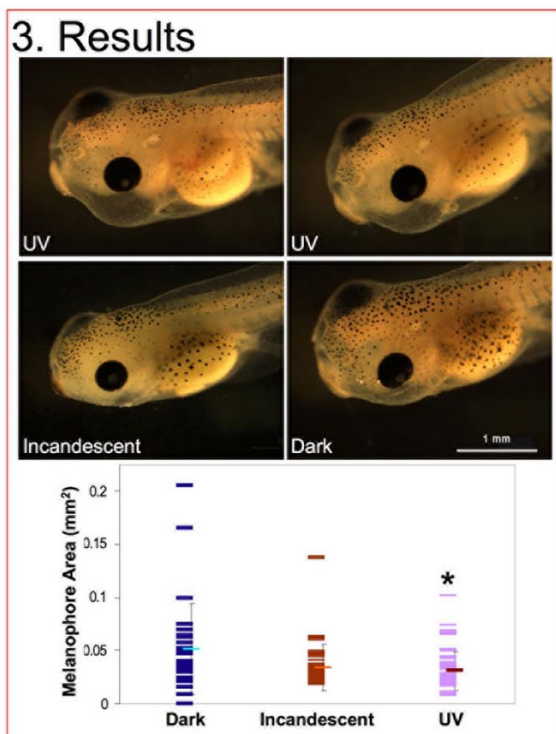

Embryo melanophore area on gut varies with raising in UV light, incandescent light, and darkness. Representative images show low melanophore area in UV-raised tadpoles to high melanophore area in dark-raised tadpoles. Graph shows that UV light-raised tadpoles had significantly less melanophore area than dark-raised tadpoles $(\mathrm{P}=0.043)$.
4. Results

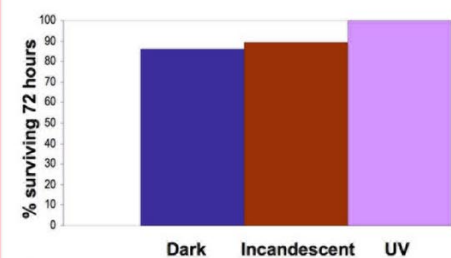

Survival Rate of Xenopus tadpoles exposed to different types of light. UV light-raised tadpoles had similar survival rates to incandescent or darkraised tadpoles, indicating that UV light does not affect death rate in Xenopus before stage 42 .

\section{Conclusions}

-Xenopus produce smaller melanophore area in UV light than in incandescent light before stage 42 of development

-Xenopus embryos do not appear to have evolved a melanophore-related mechanism to combat UV rays

- Low melanophore area in UV light-raised tadpoles did not appear to affect their survival

UV light exposure decreases melanophore area in Xenopus laevis embryos

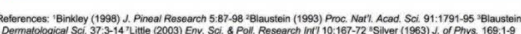

Figure 6. Poster presentations as alternatives to lab reports. (A) Student-generated mini-posters from the semi-independent research project are displayed on a lab bulletin board as a means for students to compare research results and examples of how data can be presented visually. (B) A poster detailing a student's independent research project can be easily projected onto a screen in order for students to give an oral presentation to their classmates, eliminating the cost and production time for large-format paper posters. (C) An example of an independent research project final poster that was improved through instructor comments and oral presentation to classmates. 
A pleasant and unanticipated side effect of our investment in image training occurred during the last few class periods when students discussed recent research articles on stem cells. Many of our students expressed surprise and dismay at the quality of some of the images in the articles our class discussed. Students confidently critiqued the scientific results of other researchers; they actively and skeptically compared the visual results presented in the figures with the authors' written claims. In fact, our students were eager to point out how the authors could improve their figures and how their written claims were not always well supported by their figures. We were impressed by the level of scientific understanding and reasoning the students demonstrated in conducting and communicating their own research as well as in analyzing the published research, indicating that they were able to achieve an advanced form of learning (Bloom, 1956).

In summary, learning to communicate using images instead of or in addition to words is a powerful skill that is an essential component of a developmental biologist's toolkit and is also transferable to many other areas of communication and science. We believe the evaluative and creative levels of reasoning that students gain by developing skills in visual communication will help them communicate and evaluate claims more effectively in both academic and nonacademic aspects of their lives.

\section{ACKNOWLEDGMENTS}

We thank Julie Ruble for valuable comments on the manuscript, Chris Healey for expert technical assistance throughout the Developmental Biology course, and the Davidson College students enrolled in Bio 306 for their enthusiasm, creativity, and feedback. This work was generously supported by the Howard Hughes Medical Institute, the National Science Foundation, and Davidson College.

\section{REFERENCES}

Amato, I. (2003). Super Vision: A New View of Nature, New York: Harry N. Abrams.

Bloom, B. S. (1956). Taxonomy of Educational Objectives, Handbook I: The Cognitive Domain, New York: David McKay.

Bourley, F., and Hirsch, L. (2002). Hidden Beauty: Microworlds Revealed, New York: Harry N. Abrams.
Campbell, A. M., and Lom, B. (2006). A simple e-mail mechanism to enhance reflection, independence, and communication in young researchers. CBE Life Sci. Educ. 5, 318-322.

Chatterjee, R. (2006). 2006 visualization challenge winners. Science 313(5794), 1730-1735.

Chemotti, D. C., Davis, S. N., Cook, L. W., Willoughby, I. R., Paradise, C. J., and Lom, B. (2006). The pesticide malathion disrupts Xenopus and zebrafish embryogenesis: an investigative laboratory exercise in developmental toxicology. Bioscience J. College Biol. Teach. 32, 4-18.

Clarke, T. M. (2007). Fitting a Student Microscope with a Consumer Digital Camera. www.modernmicroscopy.com/article_pix/ 030902_coolpix/MountingConsumerCamera.pdf (accessed 29 June 2007).

Council of Science Editors (2006). CSE's White Paper on Promoting Integrity in Scientific Journal Publications. www.councilscienceeditors.org/editorial_policies/whitepaper/entire_whitepaper.pdf (accessed 29 June 2007).

Davidson, M. W., and Rill, R. L. (2003). Photomicrography: Common Ground for Science and Art. www.microscopy.fsu.edu/publications/pages/mayissue.html (accessed 29 June 2007).

Frankel, F. (1998). Envisioning science-a personal perspective. Science 280(5370), 1698-1700.

Frankel, F. (2001). Communicating science through photography. J. Chem. Educ. 78, 1312-1314.

Frankel, F. (2004). Envisioning Science: The Design and Craft of the Science Image, Cambridge, MA: MIT Press.

Frankel, F., and Whitesides, G. M. (1997). On the Surface of Things: Images of the Extraordinary in Science, San Francisco, CA: Chronicle Books.

Kramer, S., and Kunkel, D. (2001). Hidden Worlds: Looking through a Scientist's Microscope, Boston, MA: Houghton Mifflin.

Martin Microscope Company (2007). Universal Microscope Adaptors for Select Digital Cameras. www.martinmicroscope.com/ New\%20MM\%20Page.htm (accessed 29 June 2007).

Nikon (2007). Nikon Small World-Photomicrography Competition. www.nikonsmallworld.com (accessed 29 June 2007).

Olympus (2007). Bioscapes International Digital Imaging Competition. www.olympusbioscapes.com (accessed 29 June 2007).

Pearson, H. (2005). Image manipulation: CSI: cell biology. Nature 434, 952-953.

Rossner, M., and Yamada, K. M. (2004). What's in a picture? The temptation of image manipulation. J. Cell Biol. 166(1), 11-15.

Softpedia (2008). Download ImageJ 1.3.9p for Mac. mac.softpedia. com/get/Graphics/ImageJ.shtml (accessed 23 January 2008). 